\title{
Neurosurgery Apps: Novel Knowledge Boosters
}

\author{
Nöroșirürji Uygulamaları: Yeni Bilgi Arturıcılar
}

Manjul TRIPATHI, Rama Chandra DEO, Vinkle SRIVASTAV, Britty BABY, Ramandeep SINGH, Natesan DAMODARAN, Ashish SURI

All India Institute of Medical Sciences, Department of Neurosurgery, New Delbi, India

Corresponding Author: Ashish SURI / E-mail: surineuro@gmail.com

\begin{abstract}
AIM: The utilization of technology for purpose of imparting knowledge, especially in high-end branches like neurosurgery, has gained prominence in the contemporary academic scenario. The technological advancements have brought about outstanding transformation to education and patient care. The connectivity through smartphone applications (apps) has transcended the spatial and temporal limitations, thereby enabling easy access to virtually infinite storehouse of knowledge. Although there are numerous neurosurgery related apps, yet there is still a dearth of quality apps that may serve the purpose.

MATERIAL and METHODS: Relevant apps were searched and evaluated on PlayStore and Apple App store, based on their content, user interface, performance, and utility in routine practice and compared with their cost, size and popularity. They were categorized into apps related to textbooks, scoring systems, patient education, operative procedures, blogs, journals, conferences and promotional.

RESULTS: 159 relevant apps were hosted on App stores; $54.7 \%$ apps were free of cost. "Neuromind" was the most downloaded app because of its simplicity, free access and applicability. Students and practitioners prefer various apps linked to scoring systems, textbooks and operative illustrations. Apps have helped patients in better understanding of their diseases and management options.

CONCLUSION: Development of web-based technologies has divided medical professionals into traditional and modernized learners. Mobile apps permit knowledge to be structured visually to facilitate its easy diffusion in the peer community. A technologically demanding branch like Neurosurgery inevitably needs innovative, cost effective apps with trust worthy content. Relevant apps have a high potential to be used as an excellent resource for effectual neurosurgical education beyond the limitations of time and place.
\end{abstract}

KEYWORDS: Smartphone, Neurosurgery apps, Education, PlayStore, App store

öz

AMAÇ: Özellikle nöroşirürji gibi üst düzey dallarda bilgi vermek için teknoloji kullanımı günümüzdeki akademik ortam içinde önem kazanmıştır. Teknolojik gelişmeler eğitim ve hasta bakımı açısından olağanüstü bir dönüşüm geçirmiştir. Akıllı telefon uygulamaları (app'ler) yoluyla bağlanabilirlik, uzaysal ve zamansal sınırlamaları aşmış ve hemen hemen sonsuz bir bilgi ambarına kolay erişimi mümkün kılmıştır. Şu anda çok sayıda nöroşirürji uygulaması olsa da işe yarayacak kaliteli uygulama sıkıntısı vardır.

YÖNTEM ve GEREÇLER: İlgili uygulamalar, PlayStore ve Apple App mağazasında araştırılmış ve içeriği, kullanıcı arayüzü, performansı ve rutin uygulamadaki faydası açısından değerlendirilip maliyet, büyüklük ve popülerlik açısından karşılaştırılmışlardır. Bunlar kitaplar, puanlama sistemleri, hasta eğitimi, operatif işlemler, bloglar, dergiler, konferanslar ve promosyonel uygulamalar şeklinde sınıflandırılmışlardır.

BULGULAR: App mağazalarında 159 ilgili uygulama bulunmuş ve bunların \%54,7'sinin ücretsiz olduğu izlenmiştir. Basitliği kolay erişimi ve uygulanabilirliği açısından en çok indirilen uygulama "Neuromind" olmuştur. Öğrenciler ve uygulayıcılar puanlama sistemleri, kitaplar ve ameliyat çizimleriyle bağlantılı çeşitli uygulamaları tercih etmektedir. Uygulamalar hastaların hastalıkları ve tedavi seçeneklerini daha iyi anlamalarına yardımcı olmuştur.

SONUÇ: Web tabanlı teknolojilerin gelişmesi tıp uzmanlarını geleneksel ve modern öğrenciler olarak bölmüştür. Mobil uygulamalar bilginin bilimsel toplulukta kolay dağılmasını sağlayacak şekilde görsel olarak yapılandırılmasını mümkün kılmaktadır. Nöroşirürji gibi teknolojik açıdan zorlu bir dal tabii ki güvenilir içerikli yenilikçi ve maliyet etkin uygulamalar gerektirecektir. İlgili uygulamaların zaman ve yer sınrılamalarının ötesinde etkili nöroşirürji eğitimi açısından mükemmel bir kaynak olarak kullanılmak üzere yüksek potansiyeli vardır.

ANAHTAR SÖZCÜKLER: Akıllı telefon, Nöroşirürji uygulamaları, Eğitim, PlayStore, App Store

ABBREVIATIONS: 2D: 2 dimensional, 3D: 3 dimensional, Apps: applications, F: free, .flv: flash video, GB: gigabyte; HTML: hypertext markup language, iOS: iPhone operating system, Kb: kilobyte, MB: megabyte, .mpeg: moving picture expert group, QR: quick response, UPC: universal product code, USD: United States Dollar 


\section{INTRODUCTION}

The $21^{\text {st }}$ century has been fortunate to witness the greatest and unanticipated transformation in the domain of communication modalities that is manifested in widespread usage of mobile communication devices and smartphone applications (apps). Though primarily introduced for entertainment, these apps have expanded into every aspect of life and productively into education and health care. The introduction of apps has brought world from screens to "palms". Every smartphone platform like Android (PlayStore), iOS (App Store), Windows Phone and Blackberry hosts variety of apps, which link to medical education.

Due to financial and regional constraints, it becomes challenging to provide an opinion on the preferred mobile operating system (OS) among neurosurgeons. Android-based smartphone and devices occupy $68.4 \%$ of mobile phone market owing to its popularity in Asian countries. On the other hand, iOS is popular in American continent and Australia, occupying $19.4 \%$ of global mobile market (6). It is difficult to find useful app among the wide cafeteria choices available. A critical analysis of these apps leaves one with an unsatisfactory note (15). These apps impart information regarding diseases and health care to patients, whereas provides guidelines for patient examination, diagnosis, management and operative procedures to neurosurgeons.

The ever-increasing number of apps and their expedient availability has added more confusion. It is difficult to choose the desired app providing relevant and updated information. Considering these difficulties, it is undeniable that there exists a dire need of a comprehensive and an all-inclusive review of available apps, which can modestly serve in guiding the community to sort out appropriate apps of their utility from the pandemonium of app markets.

\section{MATERIAL and METHODS}

Apps were searched on PlayStore (Android market) and App Store (iOS market) using the phrases "Neurosurgery", "Neurological Surgery", "Neuro Surgery", "Spine", "Neurosurgical Operations" and reviewed in April 2014. These were further evaluated on pre-defined parameters of Android versus iOS, free versus paid, cost, developer ratings, installations, synchronous versus asynchronous content (on the number of devices; data available only for Android devices), size, content user ratings, update history, and reviewers' comments. All free apps were individually evaluated after installing them over the relevant operating systems, to rate their content and performance. The paid apps were also evaluated on the basis of their performance with trial versions, when available or on the extensive customer review.

The number of installations of an app is an indirect evidence of its acceptance and popularity among users (13). After downloading an app, users rate and comment on the them (12). Any new user may view the average user ratings of these apps on the statistics page in 'Google Play Developer Console'. At the time of publishing an app, developer provides a matu- rity rating for a selected group of users. It should be followed according to 'Google Play instructions' (1).

In App store, user ratings are not available and one has to go through the content and user interface for deciding the suitability of the app. Developer ratings in iOS are categorized in $4+, 9+, 12+$ and $17+$, as per the viewer age suitability for the content (16). Software version is the process of assigning numbers to unique states of application, which also provide details of updates (17).

\section{RESULTS}

Total 159 apps were considered relevant on both the markets. A large number of apps were not directly related to neurosurgery (viz. chiropractic maneuvers, acupuncture, fiction novels, stories, blogs etc.) hence were omitted. Spine category hosts maximum number of irrelevant apps in both markets, adding confusion to the user. The phrase "Neurological surgery" provided least number of relevant apps (Table I, II). 101/159 (63.5\%) was available in Apple stores and rest $(58=36.5 \%)$ in PlayStore. Overall, neurosurgery apps are quite less in number as compared to apps directed to general medical practice like 'Calculate by QXMD' rated 4.6/5 by 1769 users (4). The appropriate apps were further evaluated and compared on pre-defined criteria (Table III, IV).

\section{Application Category}

Apps were further categorized into seven groups namely score calculators, clinical practice, operative procedures, journals, conferences, promotional, and interactive forums / blogs. Some apps catered to other sub-specialties like neuropsychiatry, neurology (e.g. eHand Neurology) and neuro-critical care (e.g. Neurointensive Care guide).

\section{Free Versus Paid}

$87 / 159$ (54.7\%) apps were free of cost. Interestingly, PlayStore hosts 1.34 times more free apps than paid apps. The costliest apps on App Store were iSpineOperations and iSpinePainManagement, each at USD75. The priciest one on PlayStore was 'Neurosurgery: 1000MCQ', costing USD 14. Most of the paid apps available on PlayStore ranged between USD 0.7 to 4.5 while on App Store between USD 0.7 to 7.7 (Table III, IV).

\section{Size of App}

Size of an app is expressed in standard formats of kilobyte $(k B)$, megabyte (MB) and gigabyte (GB). In view of the limitations posed by operating systems, most of the apps were in the size range of 1-30 MB. However, few apps were considerably large in size such as iSpineCare (1.7GB) and Dynamic Spine (1.5GB) running on iOS based systems (Table III,IV). Interestingly, the costliest apps were neither largest nor most popular. There was no correlation between size, cost and quality of an app.

\section{DISCUSSION}

\section{Most Popular Apps}

With each passing day, new medical apps are added to the 
Table I: Recommended App on App Store and PlayStore

\begin{tabular}{l|l|l}
\hline Category & App Store & Playstore \\
\hline Clinical Practice App & SOAP For iPad, AANS Grand Rounds, & $\begin{array}{l}\text { Neurosurgery Survival Guide, AANS Grand } \\
\text { Rounds, Neuroslice }\end{array}$ \\
\hline Procedural App & iSpineCare, Neurosurgery Procedural Atlas, & Craniotomy \\
\hline Score and Calculators & Neuromind, Neurotool Kit & Neuromind, NIHSS, Glasgow Coma Scale Free \\
\hline Journal & SNI, JNS, Neurosurgery & SNI, JNS \\
\hline Promotional App & Neuro Helsinki & -
\end{tabular}

Table II: Neurosurgical Blogs Accessible with Respective Apps

App provider
Neurocirurgia Brasil
Neurosurgery
Neurosurgery (Journal)
Neurosurgery apps and decision support
Surgical Neurology International (journal)

market but apps with updated and comprehensive content are still a few. With around more than 0.2 million downloads, Neuromind tops the list among all the apps dedicated to Neurosurgery $(15,19)$ (Table III). Most of these popular apps share a unique combination of being illustrative with easy user interface and comprehensive coverage of the topics and regular revisions.

\section{Score-Calculator Apps}

Residents and trained neurosurgeons most frequently use apps related to 'scoring systems', followed by apps for anatomical illustrations (8). Most of the apps were dedicated to particular scoring system only like SimpliGlasgow or 'GCS Free app' for calculating Glasgow Coma Score and 'NHISS' app for stroke severity calculation. However, a few apps such as Neuromind systematically deal with all commonly used scoring systems in clinical practice.

\section{Clinical Practice Apps (Neuroanatomy, symptomatology, treatment guidelines etc.)}

A significant number of apps are dedicated to basic neurosurgical sciences such as neuroanatomy, patient examination and treatment, approach for management and interactive case scenarios. Some of these apps provide three dimensional (3D) visualization of cranial and spine anatomy, radiological correlation and comments over controversies of management viz. Spinal Cord Trauma (iOS) app and EASS.live app (AO Spine society app on PlayStore). Neuroslice is another very popular app among first year residents with interactive demonstration of radiological images of brain in axial, coronal and sagittal sections. Apart from these, one can also find useful apps for preparation of various board examinations e.g. AANS Board Review and Neurosurgery, 1000MCQ (Figure $1)$.
Blog

www.neurocirurgiabr.com

www.neurosurgic.com

http://neurosurgerycns.wordpress.com/

http://DigitalNeurosurgeon.com

http://surgicalneurologyinternational.com/blog/

\section{Procedural App}

Neurosurgical operations are complex and demand highest degree of accuracy in their execution. Apps dedicated to specific spine and cranial operative procedures demonstrate the intricacies of surgical steps and operative minutiae with edited video clips and narrative descriptions. Such apps (e.g. AANS Grand Round) link to operative videos library through their domain. Most of these videos are in Flash video (.flv) or 'Moving Picture Expert Group' (.mpeg) format and are hosted on popular platforms like YouTube or vimeo. It is now encouraging to find that most of these platforms are supporting their content in 'Hypertext Markup Language' (HTML5) format (e.g. SNImobile) (8). HTML5 is device independent, which makes it a potential candidate for cross platform mobile applications. There are a few apps dedicated for functional neurosurgery such as Stereocheck that helps in image based target localization and cross check of fiducial markers.

\section{Journal Related Apps}

Some popular journals have their dedicated apps for both platforms. A few journals were free access providing full content (e.g. SNI for Surgical Neurology International) while rest were providing limited content like abstract only for free users (e.g. Neurosurgery and Journal of Neurosurgery). The content of these apps was easy to navigate and downloadable providing up-to-date information about advances in Neurosurgery (Figure 2A,B).

\section{Apps in Foreign Languages}

English is the preferred language of communication used by majority of neurosurgery apps. However, a few apps are available in other popular languages as well such as Turkish, French, Spanish, Chinese and Portuguese etc. These apps 

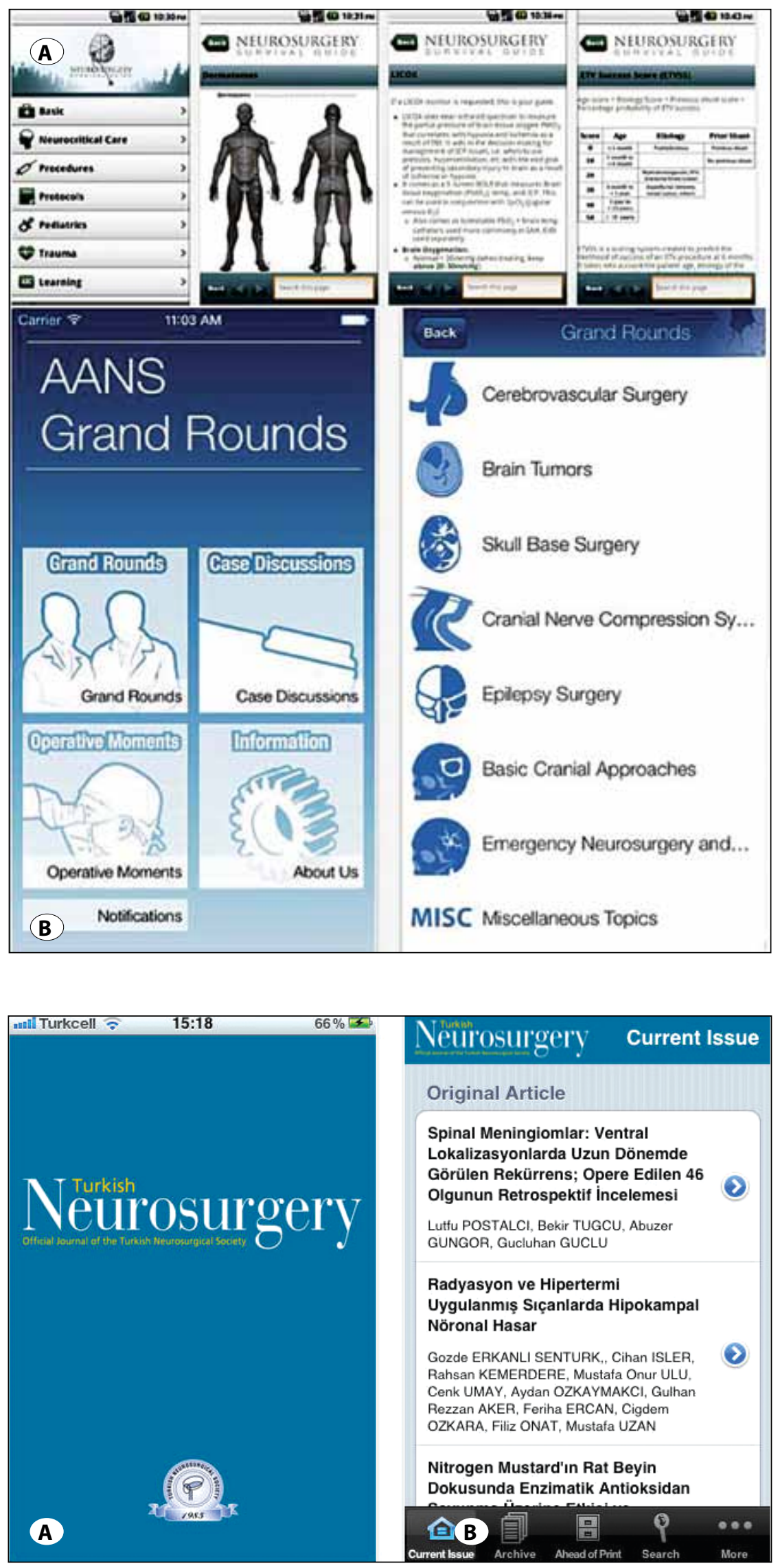

Figure 1: User interface of A) Neurosurgery Survival Guide app; B) AANS Grand Rounds.
Figure 2: A) Official app and B) User interface of Turkish Neurosurgery. 
carry their special worth in other parts of the world, where education can be imparted in their native language e.g. iNeurochirurgiae and Turkish Neurosurgery.

\section{Apps Linking to Blogs}

Blog, which is a short form of 'weblog', is a virtual diary connecting the reader to material published by a person or a community. With their subscription, one can get an update of their content on email, also known as 'feeds'. Some neurosurgical apps link them to various blogs related to their website or their social networking forums e.g. Facebook, Twitter etc. (Table II) (9).

Table III: Apps for iOS Based Smartphones (Based on Inputs from App Store)

\begin{tabular}{|c|c|c|c|c|}
\hline S No. & App Name & Cost (USD*) & Version & Size (MB) \\
\hline \multicolumn{5}{|c|}{ Category: Clinical Practice App } \\
\hline 1 & @Hand:Treatment Strategies in Neurology & 33.84 & 1.0 & 2.1 \\
\hline 2 & 1000 Neurology Dictionary and Glossary of Terms & 2.61 & 1.7 & 24.7 \\
\hline 3 & 3D Human Spine & 0.84 & 1.0 & 34.9 \\
\hline 4 & 3D Human Spine HD & 2.61 & 1.0 & 24.0 \\
\hline 5 & 3D Medical Human Spine HD & 2.61 & 1.01 & 24.8 \\
\hline 6 & 3D Medical Human Vertebral Column HD & 2.61 & 1.0 & 26.2 \\
\hline 7 & 3D Nervous System & 0.84 & 1.0 & 43.1 \\
\hline 8 & 3D Neuro System & 12.15 & 1.2 & 24.6 \\
\hline 9 & 3D Pelvis Spine Pins & 0.84 & 1.0 & 36.7 \\
\hline 10 & 3D Spine Anatomy & 0.84 & 1.0 & 37.3 \\
\hline 11 & AANS Grand Round & $\mathrm{F}$ & 2.31 & 10.8 \\
\hline 12 & All Spine Disorders & 1.69 & 1.0 & 5.8 \\
\hline 13 & All Visibility Spine & 0.84 & 1.0 & 34.9 \\
\hline 14 & Anatomy Spine & 0.84 & 1.0 & 55.9 \\
\hline 15 & Axis Atlas and Sacrum 3D & 0.84 & 1.0 & 43.6 \\
\hline 16 & Build-A-Brain Explorer & 1.69 & 1.0 & 52 \\
\hline 17 & Dynamic Spine & 16.9 & 1.0 & $1.5 \mathrm{~GB}$ \\
\hline 18 & eHand: Neurology & 33.84 & 1.0 .0 & 1.9 \\
\hline 19 & iCranialNerves & 6.92 & 1.1 & 189 \\
\hline 20 & iNeuro & 6.92 & 1.1 & 144 \\
\hline 21 & iSpinePainManagement & 75.38 & 1.2 .1 & 968 \\
\hline 22 & MHSI & $\mathrm{F}$ & 1.3 & 13.8 \\
\hline 23 & mLumbarAnatomy & 2.61 & 1.3.1 & 19.6 \\
\hline 24 & mLumbarDegenerative DiscDisease & 1.69 & 1.3 .1 & 58.2 \\
\hline 25 & mLumbarDiscHerniation & 1.69 & 1.3.1 & 53.1 \\
\hline 26 & Neurointensive Care Guide & 4.15 & 1.0 .3 & 3.9 \\
\hline 27 & Neurology Multilingual & 0.84 & 1.1 & 1.8 \\
\hline 28 & Neurorad & 16.9 & 2.1 & 104 \\
\hline 29 & Neuroradiology Multilingual & 0.84 & 1.1 & 5.8 \\
\hline 30 & Neurosurgery: Conditions \& Treatment & 0.84 & 1.3 & 14.5 \\
\hline 31 & Oxford Handbook of Neurology & 41.5 & 1.5 .0 & 9.3 \\
\hline 32 & Pocket Brain & 8.46 & 2.4 & 132 \\
\hline 33 & SANS Boards & 33.84 & 1.0 .2 & 4.5 \\
\hline 34 & SOAP For iPad & 41.5 & 1.1 & 3.9 \\
\hline 35 & SOAP Lite & 0.84 & 1.1 & 3.7 \\
\hline 36 & Spinal Cord Trauma & 4.15 & 1.0 & 9.3 \\
\hline 37 & Spine Care & $\mathrm{F}$ & 2.0 & 11.2 \\
\hline
\end{tabular}


Table III: Cont.

\begin{tabular}{|c|c|c|c|c|}
\hline 38 & Spine Conditions \& Treatment & 1.69 & 1.0 & 12.1 \\
\hline 39 & Spine Decide Patient Education for Healthcare & $\mathrm{F}$ & 4.0.2 & 137 \\
\hline 40 & Spine Glossary & 1.69 & 1.0 & 1.5 \\
\hline 41 & Spine HD & 2.61 & 1.0 & 59.6 \\
\hline 42 & Spine Imaging Case Review & 16.9 & 1.0 & 85.2 \\
\hline 43 & Spine Pro III & 8.46 & 3.7 & $1.4 \mathrm{~GB}$ \\
\hline 44 & SpineEffects & $\mathrm{F}$ & 1.0 & 35.8 \\
\hline 45 & The Complete Human Brain PRO & 41.5 & 1.0 & 22.9 \\
\hline 46 & The Kinetic Spine & $\mathrm{F}$ & 1.0 & 24 \\
\hline 47 & Vertebra Structure 3D & 0.84 & 1.0 & 43.2 \\
\hline 48 & SLIC & $\mathrm{F}$ & 2.0 & 5.0 \\
\hline \multicolumn{5}{|c|}{ Category: Neurosurgical Procedure } \\
\hline 49 & iNeuroMobile & $\mathrm{F}$ & 1.0.2 & 23.4 \\
\hline 50 & iSpineCare & 59.99 & 1.4 .1 & $1.7 \mathrm{~GB}$ \\
\hline 51 & iSpineOperations & 75.38 & 1.3 .2 & 1.3 \\
\hline 52 & mLumbarLaminectomy & 2.61 & 1.2.1 & 66.5 \\
\hline 53 & mLumbarMicroDiscectomy & 2.61 & 1.2 .1 & 71.2 \\
\hline 54 & mLumbarPosteriorInterbodyFusionCage & 2.61 & 1.2 .1 & 173 \\
\hline 55 & NeuroClin Instruments & $\mathrm{F}$ & 1.0 & 22.3 \\
\hline 56 & Neurosurgery: A Procedural Atlas & 2.61 & 1.0 & 6.2 \\
\hline 57 & OOTApp & $\mathrm{F}$ & 1.0 .0 & 8.4 \\
\hline 58 & StereoCheck & 8.46 & 1.3 & 1.2 \\
\hline 59 & Thomale Guide Application & $\mathrm{F}$ & 1.1.1 & 14.2 \\
\hline 60 & VCath & $\mathrm{F}$ & 1.02 & 26.7 \\
\hline \multicolumn{5}{|c|}{ Category: Score Calculators } \\
\hline 61 & Neuro Toolkit & 2.61 & 2.999 & 4.5 \\
\hline 62 & Neuromind & $\mathrm{F}$ & 2.2 & 13.4 \\
\hline 63 & SimpliGlasgow & 0.84 & 2.0 & 52.8 \\
\hline 64 & SLIC & $\mathrm{F}$ & 2.0 & 4.7 \\
\hline \multicolumn{5}{|c|}{ Category: Neurosurgery Journals } \\
\hline 65 & GIS & $\mathrm{F}$ & 1.2 & 2.3 \\
\hline 66 & JNS App & $\mathrm{F}$ & 2.0 & 4.7 \\
\hline 67 & Journal of Spinal Disorders & $\mathrm{F}$ & 1.1 & 7.5 \\
\hline 68 & Neurosurgery & $\mathrm{F}$ & 1.3 & 5.2 \\
\hline 69 & Spinal News International & $\mathrm{F}$ & 1.0 & 12.9 \\
\hline 70 & Spine & $\mathrm{F}$ & 1.2 & 9.4 \\
\hline 71 & Turkish Neurosurgery & $\mathrm{F}$ & 6.4 & 2.7 \\
\hline 72 & The Spine Journal & $\mathrm{F}$ & 3.8 .10 & 5.5 \\
\hline 73 & SNI International & $\mathrm{F}$ & 1.1 & 4.0 \\
\hline \multicolumn{5}{|c|}{ Category: Neurosurgery Promotional } \\
\hline 74 & Aesculap Spine Cervical & $\mathrm{F}$ & 1.6.7 & 13.3 \\
\hline 75 & Aesculap Spine MIS Thoracolumbar & $\mathrm{F}$ & 1.7.4 & 8.1 \\
\hline 76 & AAOS eBooks & $\mathrm{F}$ & 3.3 .3 & 5.3 \\
\hline 77 & Aesculap Neuroendoscopy & $\mathrm{F}$ & 1.6 .7 & 15.1 \\
\hline
\end{tabular}


Table III: Cont.

\begin{tabular}{|c|c|c|c|c|}
\hline 78 & AuroraSpine & $\mathrm{F}$ & 1.1 & 20.8 \\
\hline 79 & BASS Access & $\mathrm{F}$ & 1.2 & 4.6 \\
\hline 80 & coflex & $\mathrm{F}$ & 3.0 .0 & 12.1 \\
\hline 81 & DePuy Synthes eModels & $\mathrm{F}$ & $A D$ & 20.6 \\
\hline 82 & DePuySynthes International & $\mathrm{F}$ & 3.01 .1 & 3.7 \\
\hline 83 & mLumbarPosteriorInterbodyFusionCage & 2.61 & 1.2 .1 & 173 \\
\hline 84 & Paradigm Spine & $\mathrm{F}$ & 3.0 .0 & 11.6 \\
\hline 85 & SI-LOK & $\mathrm{F}$ & 1.0 & 24.4 \\
\hline 86 & Stryker IVS for iPad & $\mathrm{F}$ & 1.1 .1 & 777 \\
\hline 87 & Thieme Bookshelf & $\mathrm{F}$ & 3.3 .1 & 3.4 \\
\hline 88 & Yellosteps 3D Viewer & $\mathrm{F}$ & 1.03 & 17 \\
\hline 89 & Neuro Helsinki & $\mathrm{F}$ & 1.054 & 12.6 \\
\hline \multicolumn{5}{|c|}{ Category: Neurosurgery Conferences } \\
\hline 90 & $10^{\text {th }}$ ECE, London2012 & $\mathrm{F}$ & 1.0 & 6.5 \\
\hline 91 & $30^{\text {th }}$ IEC Montreal 213 & $\mathrm{~F}$ & 2.2 .1 & 12.3 \\
\hline 92 & CNS 2013 Annual Meeting Guide & $\mathrm{F}$ & 1.6 & 38.9 \\
\hline 93 & CSRS 2013 Mobile & $\mathrm{F}$ & 4.0 & 2.0 \\
\hline 94 & DGNC 2013 & $\mathrm{~F}$ & 1.1 & 18 \\
\hline 95 & EuroSpine HD & $\mathrm{F}$ & 3.1 & 36.8 \\
\hline 96 & ISASS HD & $\mathrm{F}$ & 3.0 .2 & 21.7 \\
\hline 97 & LINNC & $\mathrm{F}$ & 3.6.0 & 6.7 \\
\hline 98 & NASS Annual Meeting 2012 & $\mathrm{~F}$ & 1.1 & 14 \\
\hline 99 & NASS Annual Meeting 2013 & $\mathrm{~F}$ & 1.4 & 2 \\
\hline 100 & SNIS 2012 HD & $\mathrm{F}$ & 2.2 & 12 \\
\hline 101 & SNIS IESC/CV Section Annual HD & $\mathrm{F}$ & 2.2 & 11.3 \\
\hline
\end{tabular}

App, application; $\boldsymbol{F}$, free of cost; $\mathbf{M B}$, megabyte; *USD, United States Dollar.

\section{Apps Linking To Conferences}

These are extremely useful apps that link to the upcoming events of Neurosurgical Societies, and provide information about schedules, lectures and map directions for that particular event. They find their special favor among the foreign delegates as they help them with roadmap, economic hotels and conveyance options, local customs and commonly used phrases in non-native lands (Table III, IV). The hosts thoughtfully provide these apps for the registered users. In an effort to continue medical education, app can be directed to the archived educational content presented and discussed during the conference otherwise, these apps loose their worth after the event, and their removal would be a responsible act by the conference organizers.

\section{Use of Quick Response (QR) code in apps}

QR code is a trademark of two-dimensional (2D) matrix barcode arranged in a square grid on a white background, which can be read by an imaging device and processed over Internet. It provides quick access, fast readability, and greater storage capacity compared to traditional standard 'Universal
Product Code (UPC)' barcodes. A few apps like SNI mobile (Figure 3) (14), and Neurosurgery are using QR codes for easy access for their published material (18).

\section{Apps With 3D Content}

Some apps (e.g. 3D Spine Anatomy, Vertebra Structure 3D) provide anatomical and procedural illustration with the help of 3D models for better understanding of the subject. Such apps are in demand but they occupy significant space on the hard discs and require advanced operating systems for smooth performance.

\section{Promotional Apps}

This interesting segment of apps is meant for advertisement

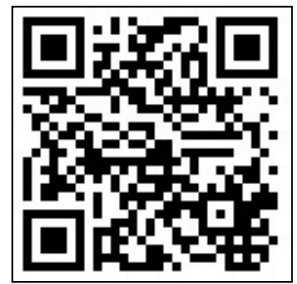

Figure 3: An example of QR code directing to Surgical Neurology International homepage $(15,19)$. 
Table IV: Apps for Android Based Smart Phones (Based on Inputs from PlayStore)

\begin{tabular}{|c|c|c|c|c|c|}
\hline S No. & App Name & Cost (USD $)$ & User Ratings & $\begin{array}{l}\text { No. of } \\
\text { Downloads }\end{array}$ & Size \\
\hline \multicolumn{6}{|c|}{ Category: Clinical Practice App } \\
\hline 1 & 3D Spine Structure & 0.97 & $0-0$ & $10-50$ & $29 \mathrm{MB}$ \\
\hline 2 & AANS Grand Rounds & $\mathrm{F}$ & $4.9 / 9$ & $1,000-5,000$ & $2.7 \mathrm{MB}$ \\
\hline 3 & Craniotomy & 3.34 & $3.85 / 7$ & $100-500$ & $12 \mathrm{MB}$ \\
\hline 4 & Deep Brain Stimulation & $\mathrm{F}$ & $0-0$ & $100-500$ & 3.5MB \\
\hline 5 & EBSS.live & $\mathrm{F}$ & $5 / 6$ & $1000-5000$ & $12 \mathrm{MB}$ \\
\hline 6 & Medical-Surgical Exam Prep & 4.14 & $0 / 0$ & $100-500$ & $552 \mathrm{kB}$ \\
\hline 7 & Medrills(Spinal Cord Injury) & 3.36 & $0-0$ & $10-50$ & $85 \mathrm{MB}$ \\
\hline 8 & MHSI & $\mathrm{F}$ & $5 / 1$ & $100-500$ & 2.6MB \\
\hline 9 & my Surgical Assistant Free & $\mathrm{F}$ & $1.8 / 4$ & $1,000-5,000$ & $739 \mathrm{kB}$ \\
\hline 10 & My SurgicalAssistant & 0.83 & $0-0$ & $10-50$ & $238 \mathrm{kB}$ \\
\hline 11 & Neuro Exam & 4.70 & $5.0 / 2$ & $100-500$ & $17 \mathrm{MB}$ \\
\hline 12 & Neuro Radiology - Brain Lite & $\mathrm{F}$ & $4.8 / 8$ & $5,000-10,000$ & 3.2MB \\
\hline 13 & Neuroinfect App & 4.17 & $5.0 / 1$ & $10-50$ & $12 \mathrm{MB}$ \\
\hline 14 & Neurolnfect App & $\mathrm{F}$ & $4.2 / 5$ & $1,000-5,000$ & $11 \mathrm{MB}$ \\
\hline 15 & Neurointensive Care Guide & 4.21 & $4.5 / 6$ & $100-500$ & 3.1MB \\
\hline 16 & NeuroRadiology - Brain & 3.42 & $3.8 / 4$ & $100-500$ & $18 \mathrm{MB}$ \\
\hline 17 & NeuroSlice & $\mathrm{F}$ & $4.5 / 97$ & $50,000-100,0000$ & 5.3MB \\
\hline 18 & Neurosurgery & 2.52 & $3.0 / 4$ & $100-500$ & $71 \mathrm{kB}$ \\
\hline 19 & Neurosurgery Conditions \& Treatment & 0.84 & $3.0 / 2$ & $50-100$ & 3.6MB \\
\hline 20 & Neurosurgery Survival Guide & 5.84 & $4.8 / 27$ & $1000-5000$ & $21 \mathrm{MB}$ \\
\hline 21 & Neurosurgery, 1000 MCQs & 14.12 & $0-0$ & $1-5$ & $12 \mathrm{MB}$ \\
\hline 22 & Quiz- Anatomy/Physiology Neurology & 4.21 & $3.5 / 4$ & $100-500$ & $713 \mathrm{kB}$ \\
\hline 23 & Skyscape (Back and Spinal cord) & 12.6 & $0-0$ & $50-100$ & $831 \mathrm{kB}$ \\
\hline 24 & Spinal Cord/ Nerve flash cards & $\mathrm{F}$ & $5 / 2$ & $500-1000$ & $5 \mathrm{MB}$ \\
\hline 25 & Spine Glossary & 0.83 & $0-0$ & $100-500$ & $1.9 \mathrm{MB}$ \\
\hline 26 & Spine MD & $\mathrm{F}$ & $0-0$ & $100-500$ & $4.3 \mathrm{MB}$ \\
\hline 27 & Surgical Assistant & 0.83 & $3.2 / 5$ & $100-500$ & $168 \mathrm{kB}$ \\
\hline 28 & Traumatic Brain Injury (TBI) & 4.21 & $5.0 / 4$ & $100-500$ & 4.1MB \\
\hline 29 & SLIC & $\mathrm{F}$ & $4.6 / 5$ & $1000-5000$ & 4.4 MB \\
\hline \multicolumn{6}{|c|}{ Category: Scores Calculators } \\
\hline 30 & Glasgow Coma Scale & 0.88 & 0.0 & $10-50$ & $199 \mathrm{kB}$ \\
\hline 31 & Glasgow Coma Scale Free & $\mathrm{F}$ & $4.5 / 42$ & $50000-100000$ & $278 \mathrm{~KB}$ \\
\hline 32 & My Neuro Torch & 0.84 & $5 / 3$ & $50-100$ & $206 \mathrm{kB}$ \\
\hline 33 & NeuroMind & $\mathrm{F}$ & $4.2 / 112$ & $10,000-50,000$ & $12 \mathrm{MB}$ \\
\hline 34 & NIHSS & $\mathrm{F}$ & $4.7 / 55$ & $10,000-50,000$ & $344 \mathrm{kB}$ \\
\hline 35 & NIHSS-PRO & 0.82 & $5.0 / 3$ & $50-100$ & $1.0 \mathrm{MB}$ \\
\hline \multicolumn{6}{|c|}{ Category: Neurosurgery Journals } \\
\hline 36 & Journal of Neurosurgery Online & $\mathrm{F}$ & $4.5 / 4$ & $1,000-5,000$ & $2.2 \mathrm{MB}$ \\
\hline 37 & Neurocritical Care & $\mathrm{F}$ & $5 / 1$ & $100-500$ & $3.6 \mathrm{MB}$ \\
\hline 38 & SNI Mobile & $\mathrm{F}$ & $3.6 / 7$ & $1,000-5,000$ & $3.1 \mathrm{MB}$ \\
\hline 39 & Turkish Neurosurgery & $\mathrm{F}$ & $3 / 3$ & $50-1,00$ & $358 \mathrm{kB}$ \\
\hline
\end{tabular}


Table IV: Cont.

\begin{tabular}{|c|c|c|c|c|c|}
\hline \multicolumn{6}{|c|}{ Category: Neurosurgery Promotional } \\
\hline 40 & Neurosurgery - CIMS Hospital & $\mathrm{F}$ & $0-0$ & $100-500$ & $2.2 \mathrm{MB}$ \\
\hline 41 & SANS & $\mathrm{F}$ & $0-0$ & $10-50$ & $5.6 \mathrm{MB}$ \\
\hline 42 & Spine and Health Institute & $\mathrm{F}$ & $0-0$ & $50-100$ & $376 \mathrm{kB}$ \\
\hline 43 & Valley Spine Care & $\mathrm{F}$ & $0-0$ & $10-50$ & $2.6 \mathrm{MB}$ \\
\hline 44 & VTIConnect & $\mathrm{F}$ & $5 / 4$ & $100-500$ & $2.6 \mathrm{MB}$ \\
\hline \multicolumn{6}{|c|}{ Category: Apps Connecting to Blogs } \\
\hline 45 & Chiari Malformation Awareness & $\mathrm{F}$ & $1 / 1$ & $100-500$ & $2.9 \mathrm{MB}$ \\
\hline 46 & Headache App & $\mathrm{F}$ & $3.6 / 24$ & $1,000-5,000$ & $1.9 \mathrm{MB}$ \\
\hline 47 & Neurosurgery & $\mathrm{F}$ & $4 / 4$ & $1,000-5,000$ & $914 \mathrm{kB}$ \\
\hline 48 & Neurosurgery Blog & $\mathrm{F}$ & $5 / 7$ & $1,000-5,000$ & $1.3 \mathrm{MB}$ \\
\hline \multicolumn{6}{|c|}{ Category: Neurosurgery Conferences } \\
\hline 49 & AES AnnuMtg & $\mathrm{F}$ & $1.6 / 5$ & $100-500$ & 3.6MB \\
\hline 50 & DGNC 2013 & $\mathrm{~F}$ & $0-0$ & $100-500$ & $6.5 \mathrm{MB}$ \\
\hline 51 & iSASS & $\mathrm{F}$ & $0-0$ & $100-500$ & 7.1MB \\
\hline 52 & JNS 2013 & $\mathrm{~F}$ & $0-0$ & $100-500$ & $7.4 \mathrm{MB}$ \\
\hline 53 & LINNC & $\mathrm{F}$ & $5 / 1$ & $50-100$ & 3.6MB \\
\hline 54 & NCS 2013 Annual Meeting & $\mathrm{F}$ & $0-0$ & $10-50$ & $2.0 \mathrm{MB}$ \\
\hline 55 & SNIS 10th Annual Meeting & $\mathrm{F}$ & $0-0$ & $10-50$ & $3.5 \mathrm{MB}$ \\
\hline 56 & SNIS 2012 & $\mathrm{~F}$ & $5 / 1$ & $100-500$ & 4.5MB \\
\hline 57 & SNIS IESC/CV Section Annual & $\mathrm{F}$ & $0-0$ & $50-100$ & $2.7 \mathrm{MB}$ \\
\hline 58 & WCN 2013 & $\mathrm{~F}$ & $0-0$ & $1-5$ & $8.5 \mathrm{MB}$ \\
\hline
\end{tabular}

App, application; $\boldsymbol{F}$, free of cost; GB, gigabyte; $\mathbf{k B}$, kilobyte; $\mathbf{M B}$, megabyte; *USD, United States Dollar.

purposes. Their utility varies from hospital advertisement, product information for implants (e.g. DePuySynthes International) to even appointment of a neurosurgeon. Braun (Aesculap) has separate apps for spinal and Neuroendoscopy procedures featuring operative videos involving their products and their appropriate usage. Obviously, these are free of cost with low popularity level (Table III, IV).

\section{Patient Education Apps}

A significant number of apps were available for illustrating diseases and their proposed treatment in easily understandable language for patients and relatives. Some of these apps are used by neurosurgeons for explaining patients about their disease with anatomical 2D or 3D illustrations and treatment details (Figure 4). Though such apps e.g. iSpineCare can be helpful for anyone, who wants to know about their disease, their high cost might pose a constraint to patients (Table III, IV).

\section{Relevance of Neurosurgery App to Residents in Developing Nations}

All smart apps need compatible devices for their smooth performance. Android based devices either mobile phones or pads are quite cheap (USD46) in comparison to iOS based devices (USD310). The price limitation makes Android-based devices more popular among developing countries. On an average, 3-5 neurosurgery apps (related to neurosurgery scoring systems, textbooks, operative procedures, interactive platforms and patient illustrations) are required for routine neurosurgery practice. Limited Internet access, poor technology and user support acts as a barrier for efficient usage. A certain number of relevant apps need to be purchased. This evaluation highlights the need for cheaper apps, as they are primarily dedicated for education and health care. We believe that there should be no time, place or monetary constraints for any learner of any class or creed that could limit their potential because of unavailability of learning material. Again we cannot put any limitation to the extent of knowledge one seeks at any level.

\section{Do Smartphones Transmit Only Voice?}

Though these "smart" devices (mobile phone and pads) have proven their worth for clinical communication and knowledge dispersion, few new threats have arisen because of their widespread use by clinicians. More than 20 studies have demonstrated that mobile communication devices are notoriously bugged with infective microorganisms sufficient to cause nosocomial infections $(2,3,11)$. Smartphones being used around 27.5 times per day, stands out to be one of the most common fomite in hospital premises (20). According to 


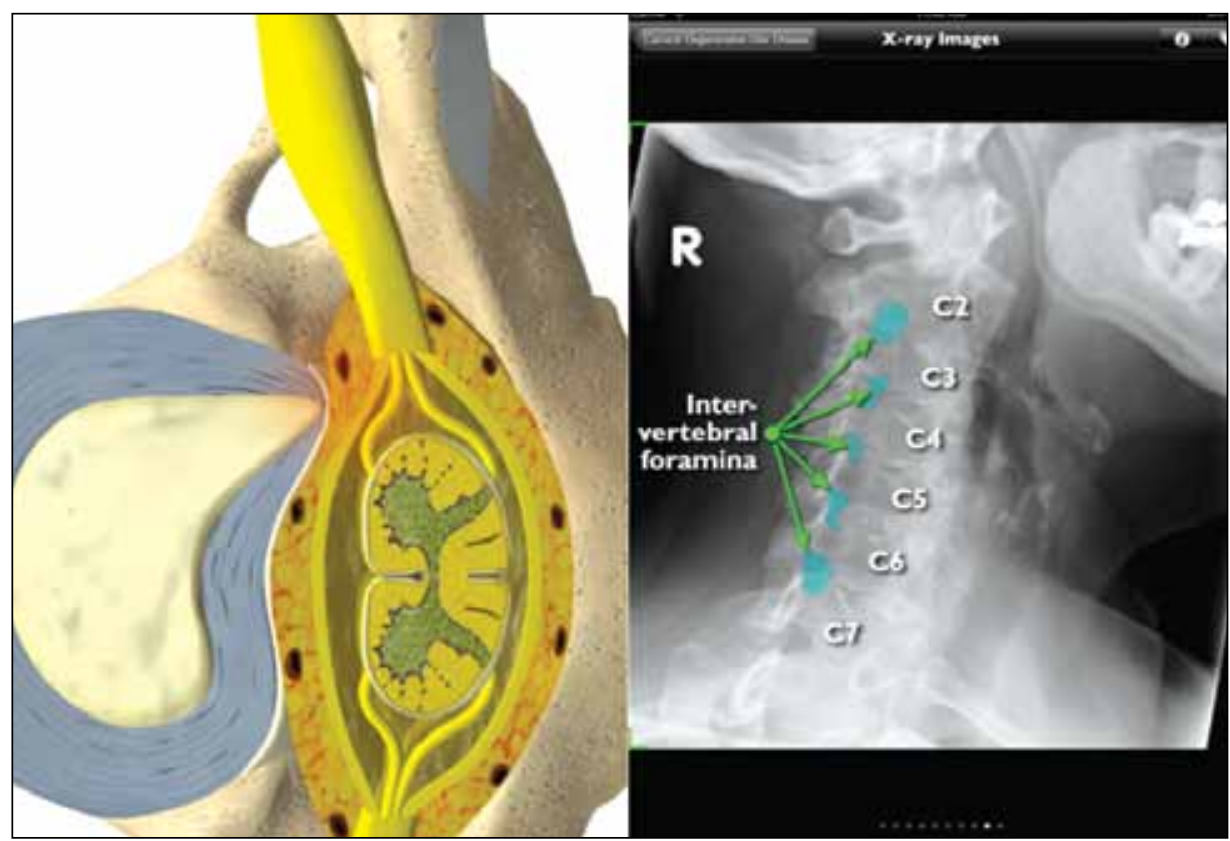

Figure 4: Snapshot of iSpineCare app showing intervertebral disc herniation causing nerve root compression. self-reports, smartphones were used during rounds for patient care ( $85 \%$ residents, $48 \%$ faculty), reading/responding to personal texts/e-mails ( $37 \%$ residents, $12 \%$ faculty), and other non-patient care uses ( $15 \%$ residents, $0 \%$ faculty). This not only interrupts the flow of information but also distracts the fellow trainees and faculties $(5,6)$ These studies warrant a need for developing 'smartphone policy' for their usage in hospital premises.

\section{CONCLUSION}

The transition of reading material from leaves to smartphones is as amazing as the evolution of mankind. With the current evaluation, we are able to suggest that good quality learning material compatible with an app is still sparse over the web. Apps with confusing similar names providing irrelevant nonreviewed data are rampant. Cheaper apps are the need of the day, as cost constraints should not be a barrier for effectual educational transfer. Any preferred app should have features of good user interface, 3D graphic content, small size, compatibility with offline usage, synchronous content support and being free of cost. Neuromind, AANS Grand Rounds, Neurosurgery Survival Guide, iSpineCare and Neuroslice are the recommended apps for routine clinical and operative practice (Table IV). There is an earnest need for a dedicated and authentic "Scholarly Search" for apps as well, directing users to quality content. Unsupervised web based learning carries a significant risk for enthusiastic learners who may not be able to access this burgeoning array of educational materials (10). Otherwise there will still be the question "Have smart apps really made neurosurgeons smarter?"

\section{ACKNOWLEDGEMENTS}

We sincerely thank Mr. Vinay Dubey for manuscript formatting. We are thankful to Mr. Subhas Bora, Mr. Ajab Singh, Mr.
Shashi Shekhar, Mr. Akash Sharma, Mr. Gaurav Bhardwaj, Mr. Suresh Kothari, Mr. Trivendra Yadav Mr. Satish Kumar, Mr. Vikram Singh, and Mr. Ramnivas, Neurosurgery Education and Training School, All India Institute of Medical Sciences, New Delhi, India for their valuable help in preparing images and statistical analysis for the manuscript.

\section{REFERENCES}

1. Applications Content Ratings. Google Play Help. Available at: https://support.google.com/googleplay/answer/1075738? hl =en. Accessed on Dec 18, 2013

2. Brady RR, Fraser SF, Dunlop MG, Paterson-Brown S, Gibb AP: Bacterial contamination of mobile communication devices in the operative environment. J Hosp Infect 66: 397-398,2007

3. Brady RR, Verran, J, Damani, NN, Gibb AP: Review of mobile communication devices as potential reservoirs of nosocomial pathogens. J Hosp Infect 71:295-300,2009

4. Daniel J: Calculate by QxMD sets the bar high for free medical calculators. iMedicalApps. Available at: http://www. imedicalapps.com/2012/03/calculate-qxmd-sets-barhigh-free-medical-calculators/. Accessed Dec18, 2013

5. Katz-Sidlow RJ, Ludwig A, Miller S, Sidlow R: Smartphone use during inpatient attending rounds: Prevalence, patterns and potential for distraction. J Hosp Med 7: 595-599,2012

6. Koetsier J: Android captured almost $70 \%$ global smartphone market share in 2012, Apple just under 20\%. Available at: http://venturebeat.com/2013/01/28/ android-captured-almost-70-global-smartphone-marketshare-in-2012-apple-just-under-20/. Accessed Dec 18, 2013 
7. Kubben PL: Neurosurgical apps for iPhone, iPod Touch, iPad and Android. Surg Neurol Int 1:89,2010

8. Kubben PL: Neurosurgical videos going mobile. Surg Neurol Int 1: 67,2010

9. Pereira JLB, Kubben PL, Freitas de Albuquerque LA, de Carvalho GC, de Sousa AA: Blogs for neurosurgeons. Surg Neurol Int 3:62-66,2012

10. Prince NJ, Cass HD, Klaber RE: Accessing e-learning and e-resources. Med Educ 44:436-437,2010

11. Ramesh J, Carter AO: Use of mobile phones by medical staff at Queen Elizabeth Hospital, Barbados: Evidence for both benefit and harm. J Hosp Infect 70:160-165,2008

12. Ratings and Comments. Android Developer Help. Available at: https://support.google.com/googleplay/ android-developer/answer/138230?hl=en. Accessed Dec 18,2013

13. Stackoverflow. Google Play meaning of installs. Available at: http://stackoverflow.com/questions/10997767/google -play-meaning-of-installs. Accessed Dec 18, 2013
14. Surgical Neurology International: SNI QR Code. Available at:http://www.surgicalneurologyint.com/viewimage. asp?img=SurgNeurollnt_2011_2_1_104_83386_f2.jpg. Accessed Oct 31, 2013

15. Tseng J: Review of neurosurgical smartphone applications 2012. Journal MTM 1:4-10,2012

16. Wikipedia. App Store (iOS)- Application Ratings. Available at:http://en.wikipedia.org/wiki/App_Store_(iOS)\# Application_ratings. Accessed Dec 18, 2013

17. Wikipedia. Software Versioning. Available at: http:// en.wikipedia.org/wiki/Software_versioning.Accessed Dec 18, 2013

18. Wikipedia. QR Code. Available at: http://en.wikipedia.org/ wiki/QR_code. Accessed Dec 18, 2013

19. Wodajo F: Popular Neurosurgery app Neuromind updated with iPad and Android versions. Available at: http://www. imedicalapps.com/2011/03/popular-neurosurgery-appneuromind-now-available-in-ipad-and-android-versions/. Accessed Dec 18, 2013

20. Wu RC, Morra D, Quan S: The use of smartphones for clinical communication on internal medicine wards. J Hosp Med 2010. Accessed March 31, 2014 